\title{
Embedded Fuzzy Control System: Application to an Electromechanical System
}

\author{
R.E. Haber ${ }^{1,2}$, J.R. Alique ${ }^{1}$, A. Alique ${ }^{1}$, and J.E. Jiménez ${ }^{1}$ \\ ${ }^{1}$ Instituto de Automática Industrial (CSIC) \\ $\mathrm{N}^{\mathrm{o}}$-III km. 22800, La Poveda. 28500. Madrid. SPAIN. \\ rhaber@iai.csic.es \\ ${ }^{2}$ Escuela Técnica Superior \\ Ciudad Universitaria de Cantoblanco \\ Ctra. de Colmenar Viejo, km. 15. 28049 - SPAIN \\ Rodolfo.Haber@ii.uam.es
}

\begin{abstract}
Nowadays with open computerized numerical controls internal control signals can be gathered and mathematically processed by means of integrated applications. Working with a commercial open computerized numerical control, a fuzzy control system has been designed, implemented and embedded that can provide an additional optimization function for cutting speed. The results show that, at least in rough milling operations, internal signals can double as an intelligent, sensorless control system. The integration process, design steps and results of applying an embedded fuzzy control system are shown through the example of real machining operations.
\end{abstract}

\section{Introduction}

One of the main activities the manufacturing industry has to deal with is machining, a process that includes operations that range from rough milling to finishing. There is a number of angles from which to view the optimization of the machining process, angles where minimum production cost, maximum productivity and maximum profit are significant factors [1].

There are also various different ways of implementing machining process optimization. The implementation on which we will focus here attains optimal goals via automatic control of the machining process. The spectrum of "conventional" methods (so named to distinguish them from intelligent methods) available for designing control systems is enormous. In the incessant pursuit of better performance, newer approaches have been also tested such as model reference adaptive control (MRAC) [2], which incorporates an on-line estimation scheme to tune controller parameters for time-varying process dynamics. Another recently applied method is robust control based on the Quantitative Feedback Theory (QFT) [3]. The results of these tests, however, have not lived up to expectations, because all these approaches have the indispensable design requisite of an accurate (traditional) process model, 
e.g., differential equations, transfer functions and state equations. Unfortunately, accurate models of this sort cannot yet be attained for the machining process.

The complexity and uncertainty of processes like the machining process are what make what is known as intelligent systems technology a feasible option to classical control strategies. Indeed, Artificial Intelligence techniques have received considerable interest from scientific community and have been applied to machining [4]-[5]. Two interesting approaches are the neural network and expert rule based on adaptive control constraints (ACC) [6] and the evolutionary algorithms based on adaptive control optimization (ACO) approach [7].

However, the main disadvantage related with the above-mentioned approaches is that neural network and evolutionary algorithm based computation requires time, and therefore it limits the performance of the intelligent control system. Nowadays, open computerized numerical control is powerful to build up an intelligent system. However, there are constraints for real-time signal processing and to implement complex control algorithms.

On the other hand, the majority of the work in machining optimization is devoted to the issue of adaptive techniques. Adaptive controllers are highly expensive considering the time requirements because they estimate parameters on-line and adjust the controller gains accordingly. Likewise, these systems must be carefully tuned and exhibit complex, and sometimes undesirable behavior.

In order to improve machining efficiency, the current study focuses on the design and implementation of an intelligent controller in an open computerized numerical control system. From all available techniques, Fuzzy Logic (FL) is selected because it has proven useful in control and industrial engineering as a highly practical optimizing tool. To the best of our knowledge, the main advantage of the present approach includes: (i) embedded fuzzy controller in an open computerized numerical control to deal with a real life industrial process, (ii) a simple computational procedure to fulfill the time requirements, and (iii) without restrictions concerning sensor cost (sensorless application), wiring and synchronization. The results with fuzzy control strategy through actual industrial tests show a higher machining efficiency.

This paper is organized as follows. In Section 2 we present a brief study of the machining process, explaining why it is considered a complex process and setting up the milling process as a case study. In Section 3 we design the fuzzy controller to optimize the milling process. Next, in Section 4 we describe how the fuzzy controller can be embedded in open computerized numerical control, and we discuss the key design and programming stages. In Section 5 we share the experimental results and explore some comparative studies. Finally we give some concluding remarks.

\section{The Machining Process}

This section introduces the milling process, which is characterised by both nonlinear characteristics and varying process parameters. The task of controlling this complex plant is performed with the help of a fuzzy controller that will be introduced in Section 3. A panoramic view of a typical machining center is given in Figure 1. 


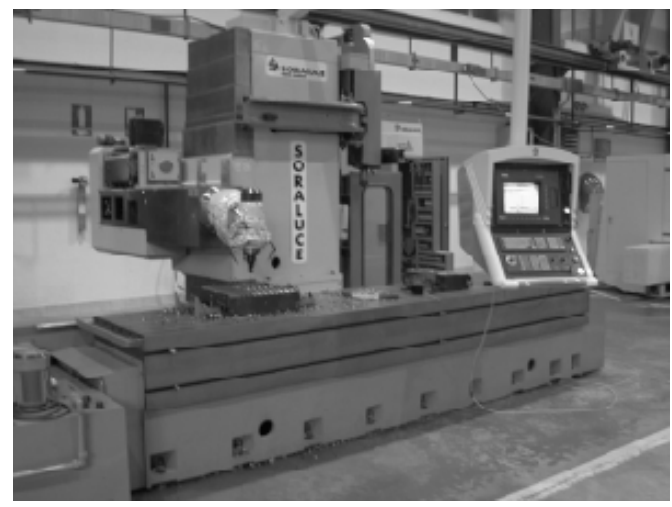

Fig. 1. Overall view of a typical machining center

The machining process, also known as the metal removal process, is widely used in manufacturing. One of the most complex of the four operations is the milling process. One important study of cutting-process monitoring and control by means of current measurement shows the feasibility of motor-current measurement for adaptive control [8]. The direct relationship between the current consumed and the cutting force is fair enough for a real industrial implementation of the control system to be made on the basis of a main spindle's drive current [9].

Nowadays the development of open control systems in the NCK offers more facilities for using digital drive signals without the need to install more sensors. Indeed, cutting processes show significant effects on drive signals such as actual drive current and drive torque. Digital drive signals have many limitations for process monitoring alone, because of the ratio between process-related components of the signal and non-process-related disturbances. However, main-spindle drive current can be used to optimize cutting speed. Signal behavior is complex because of specific design considerations such as star-triangle switching in drive configuration. Nevertheless, correcting the current offsets during the signal-processing stage (before entering the fuzzy algorithm) solves the problem.

In terms of control system design, the most important aspects are the variables, parameters and typical measures of performance that we use to characterize the system. After a preliminary study we selected: the spatial position of the cutting tool, considering the Cartesian coordinate axes $(x, y, z)[\mathrm{mm}]$, spindle speed $(s)$ [rpm], relative feed speed between tool and worktable $(f$, feedrate) $[\mathrm{mm} / \mathrm{min}]$, cutting power invested in removing metal chips from the workpiece $\left(P_{c}\right)[k W]$, current consumed in the main spindle during the removal of metal chips $\left(I_{S}\right)[\mathrm{A}]$, radial depth of cut $(a$, cutting depth) $[\mathrm{mm}]$ and cutting-tool diameter $(d)[\mathrm{mm}]$.

In order to evaluate system performance, we need to select certain suitable performance indices. The milling process basically consists of two operations, rough milling and finishing. The differences in these operations' objectives are what will decide which performance index is useful in each operation. The quality and geometric profile of the cutting surface is paramount in finishing operations, whereas the quantity of metal removed from the workpiece is the main issue in rough milling operations. This work dealt essentially with rough milling, so the main index was the metal-removal rate (MRR). 


\section{Fuzzy Logic Controller for Machining Processes}

The fuzzy controller follows rather classic lines. The controller's actual core performs on-line actions to control the feedrate. The three basic tasks known as fuzzification, decision-making and defuzzification were implemented on-line. The main design steps for this controller and their corresponding implementation were as follows:

1) Defining input and output variables, fuzzy partitioning and building the membership functions.

The input variables included in the error vector $\boldsymbol{e}$ are the current-consumed error ( $\Delta I_{S}$ in ampere) and the change in current-consumed error $\left(\Delta^{2} I_{S}\right.$ in ampere). The manipulated (action) variable we selected is the feed-rate increment ( $\Delta f$ in percentage of the initial value programmed into the computerized numerical control), whereas the spindle speed is considered constant and preset by the operator.

$$
\boldsymbol{e}^{T}=\left[K E \cdot \Delta I_{S} \quad K C E \cdot \Delta^{2} I_{S}\right] ; u=[G C \cdot \Delta f] .
$$

where $K E, K C E$ and $G C$ are scaling factors for inputs (error and change in error) and output (change in feed rate), respectively.

The fuzzy partition of universes of discourse and the creation of the rule base were drawn from the criteria of skilled operators, although we did have to apply a "cut and trial" procedure as well. Figure 2 shows the fuzzy partition thus obtained.

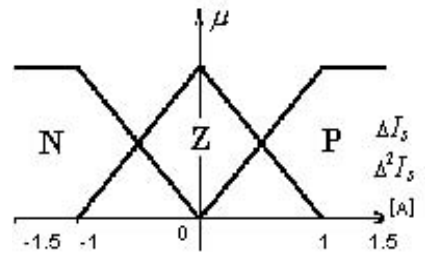

a)

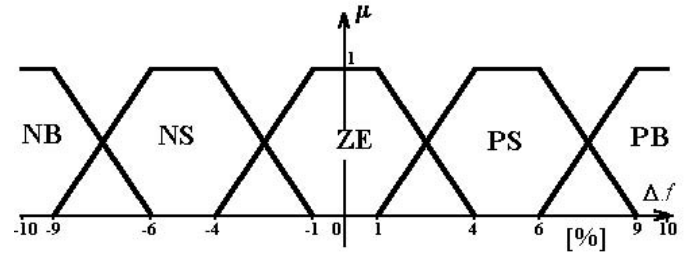

b)

Fig. 2. a) Fuzzy partitions and membership functions for (a) $\Delta I_{S}, \Delta^{2} I_{S}$ and (b) $\Delta f$

2) Constructing the Rule Base and Generating Crisp Output.

We will consider a set of rules consisting of linguistic statements that link each antecedent with its respective consequent, having, for instance, the following syntax:

IF $\Delta I_{S}$ is positive AND $\Delta^{2} I_{S}$ is positive THEN $\Delta f$ is Positive Big

The controller output is inferred by means of the compositional rule. The SupProduct compositional operator was selected for the compositional rule of inference.

$$
\mu\left(\Delta I_{S}, \Delta^{2} I_{S}, \Delta f\right)=S_{i=1}^{m \times n}\left[T_{2}\left[\mu_{\Delta I_{S_{i}}}\left(\Delta I_{S}\right), \mu_{\Delta^{2} I_{S_{i}}}\left(\Delta^{2} I_{S}\right), \mu_{\Delta f_{i}}(\Delta f)\right]\right] \text {. }
$$


where $T_{2}$ represents the algebraic product operation and $S_{1}$ represents the union operation (max), $m \times n=9$ rules. The crisp controller output, which is used to change the machine-table feedrate, is obtained by defuzzification employing the center of area (COA) method [10] defined as

$$
\Delta f^{*}=\frac{\sum_{\mathrm{i}} \mu_{R}\left(\Delta f_{i}\right) \cdot \Delta f_{i}}{\sum_{i} \mu_{R}\left(\Delta f_{i}\right)} .
$$

where $\Delta f^{*}$ is the crisp value of $\Delta f_{i}$ for a given crisp input $\left(\Delta I_{S_{i}}, \Delta^{2} I_{S_{i}}\right)$.

The crisp control action (generated at each sampling instant) defines the final actions that will be applied to the set points. The strategy used to compute $f$ determines what type of fuzzy regulator is to be used. In this case it can be a PI- FLC

$$
f *(k)=f(k-1)+\Delta f(k)
$$

or a PD- FLC

$$
f *(k)=f_{0}+\Delta f(k)
$$

Feedrate values $(f)$ were generated on-line by the controller and fed in with the set point for the spindle current $\left(I_{S r}\right)$ and measured value $\left(I_{S}\right)$ provided by the computerized numerical control from the internal spindle-drive signal, as is explained in section 4 .

The static input-output mapping can be represented by the nonlinear control surface shown in Figure 3, considering that $\Delta I_{S} \in[-1.5,1.5]$, $\Delta^{2} I_{S} \in[-1.5,1.5]$ and $\Delta f \in[-10,10]$.

These nonlinearities are essential in order to achieve good performance. Recently it has been proved that when trapezoidal membership functions are used, the resulting system is the sum of a global nonlinear controller (static part) and a local nonlinear PI controller (dynamically changing with regard to the input space) [11]. Therefore, it is expected that this kind of membership functions can be relevant for dealing with nonlinear process behavior.

\section{Open Computerized Numerical Control}

The demand for open control systems is on the rise as the result of the current need for enhanced control functionality in industry. To date, only a small group of machine-tool manufacturers has accepted adaptive controllers. One main reason for their reluctance is the need for an accurate model for calculating controller 
parameters. Additionally, adaptive controllers cannot be applied reliably to various combinations of machining processes, materials and tools without major changes being required in the control algorithm and its parameters. Some European computerized numerical control manufacturers do not include any adaptive functions in their products [12], while others offer an "AC control" whose performance is very restricted [13]. The adaptive module is rarely used in a flexible machining environment containing many types and combinations of materials and tools.

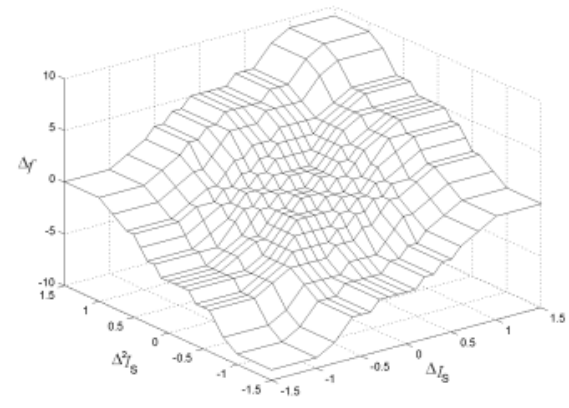

Fig. 3. Fuzzy control surface

At present two levels of opening are available from machine-tool and computerized numerical control manufacturers. The degree varies, however, depending on part involved. There are two categories, depending on how important it is from the control-system viewpoint: opening of Man/Machine Communication (MMC) and opening of the Numerical Control Kernel (NCK). The first category is the one responsible for interaction with the user (e.g., office applications can be developed using the DDE Dynamic Data Exchange protocol). Use of a bus (e.g., Multi-Point Interface bus) enables communication with low-level data. The more important level of opening from the control-system viewpoint, can be found in the NCK, where real-time critical tasks (e.g., axes control) are scheduled and performed.

\subsection{Embedding the Fuzzy Controller}

This section explains how the fuzzy controller designed in Section 3 is embedded in the open CNC. Nowadays the integration of any control system in the CNC is a complex task that requires the use of various software utilities, technologies and development tools. Three classical technologies can be used: a software-developing tool (C++, Visual Basic), an open- and real-time computerized numerical control and communications technology.

The general outline of the control system is depicted in Figure 6. First the fuzzy controller was programmed in $\mathrm{C} / \mathrm{C}++$ and compiled, and as a result a dynamic link library (DLL) was generated. Other tools were used as well. A Sun Workstation, the UNIX operating system and C++ were used to program the NCK. A PC, the WINDOWS 9X operating system and Visual Basic were used for programming the MMC. Inter-module communications between the MMC and the NCK was 
established through DDE. Finally, and for the sake of simplicity, the user interface was programmed in Visual Basic.

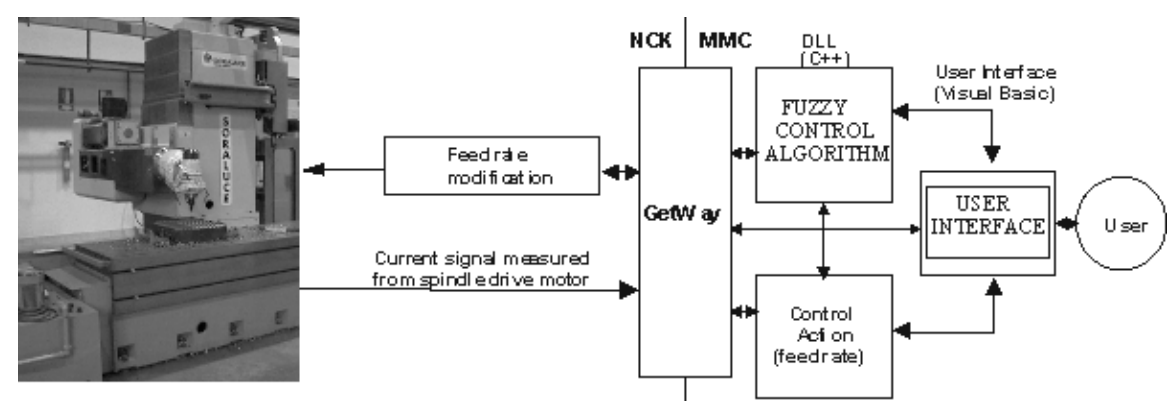

Fig. 4. Diagram of the control system.

The application was developed on the basis of a Sinumerik 840D CNC. The process of integrating the software application into the NCK involved a series of steps. The development was run at a workstation, including edition, compiling and code linking. After that, the file was transferred to the PC, where OEM software ran debugging routines. Finally, the code was copied on a PCMCIA card and inserted into the computerized numerical control [13].

An experimental control internal data-acquisition system was developed and used to record the control internal spindle-drive signal. The system enables a selected drive signal to be recorded and provides stored data on the hard drive of the SINUMERIK 840D user interface PC (MMC). Internal data-acquisition software was developed to obtain control information. The maximum sampling frequency could not be any longer than $500 \mathrm{~Hz}$, defined by the servosystems' control cycle. Therefore the acquisition-signal software works at the same time for which servosystems are configured in the computerized numerical control (i.e., $2 \mathrm{~ms}$.). The software consists of a data-acquisition module in the NCK that records the selected data into an internal buffer and an MMC background task that receives the completed measurement and stores it in the hard drive of the user-interface PC. Data transfer is performed by splitting the recorded data into a number of fragments, due to limitations in the Sinumerik 840D's file systems. The signals to be recorded (in our case the current consumed at the spindle) are configured using specially added machine data. Recording can be started and stopped either manually through an MMC application or under the numerical control program.

\section{Industrial Tests. Evaluation}

Tests were carried out in the SL400 SORALUCE machining center, which was equipped with a SIEMENS open- Sinumerik 840D. The SL400 model SORAMILL milling machine. It possesses high-precision slideways that allow all three axes to 
reach a speed of up to $15 \mathrm{~m} / \mathrm{min}$. The machine has high-rigidity, high-precision features, and the workpiece has no influence whatsoever on the moving part.

The cutting tool was a Sandvik D38 R218.19 - 2538.35 - 37.070HA (020/943) face mill $25 \mathrm{~mm}$ in diameter with four inserts of the SEKN 1204 AZ (SEK 43A, GC-A p25) type. The workpiece material used for testing was F114 quality steel. The maximum depth of cut was $20 \mathrm{~mm}$, the nominal spindle speed was $s_{0}=850 \mathrm{rpm}$, and the nominal feedrate, $f_{0}=300 \mathrm{~mm} / \mathrm{min}$. The actual dimensions of the profile were $334 \times 486(\mathrm{~mm})$. The profile is depicted in Figure 5b.

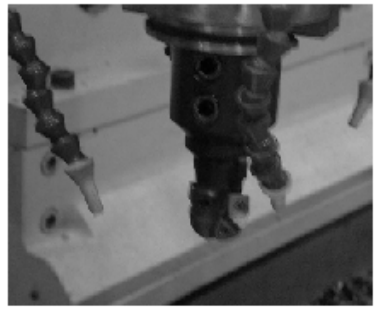

a)

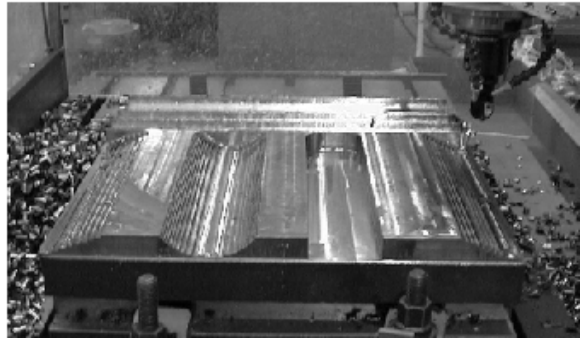

b)

Fig. 5. a) Tool used for industrial tests, b) workpiece for industrial tests

We used control internal spindle-drive signal. The processing-signal analysis was done on-line. The signal processing was centered in the correction of current offsets. The current offsets were corrected on the basis of the model that relates spindle speed vs. current. This model was created using linear regression. Moreover, further filtering was performed using the mean value of the signal in the sampling period.

The set point was estimated according to constraint given by the power available at the spindle motor, material and the tool characteristics. In order to compare the performance of the PI and PD- FLC, a reference value of 18A was set during the experiments. In the second study, the set point used was $22 \mathrm{~A}$ in order to make an appropriate comparison with a commercial product. The results of applying two fuzzy controllers (PI and PD- FLC) to mechanize an irregular profile (see Fig. 5b) are depicted in Figure 6a. The negative effect of integral components is reflected in the transient response.

An illustrative example was inserted to uphold the validity of the theoretical approaches examined in this paper. The second study compared an embedded PDFLC, the OPTIMIL [14] and the CNC working alone. The results are shown in Figure 6b. The reduction in machining time was very similar for the OPTIMIL and the embedded PD-FLC (10\%), yet the overshoot was bigger for the embedded PD-FLC $(23.7 \%)$. From the technological viewpoint, this overshoot is allowable for rough milling operations. It is important to note that in some approaches the goal of the controller design is to limit the percent overshoot to $20 \%$ [3].

Regarding OPTIMIL, when used as external equipment, requires synchronization steps and a tedious calibration procedure including a learning phase. On the other hand, the embedded PD-FLC does not require any calibration procedure or external sensors, and the application can be supplied with a computerized numerical control (CNC) alone, and no other external equipment. 


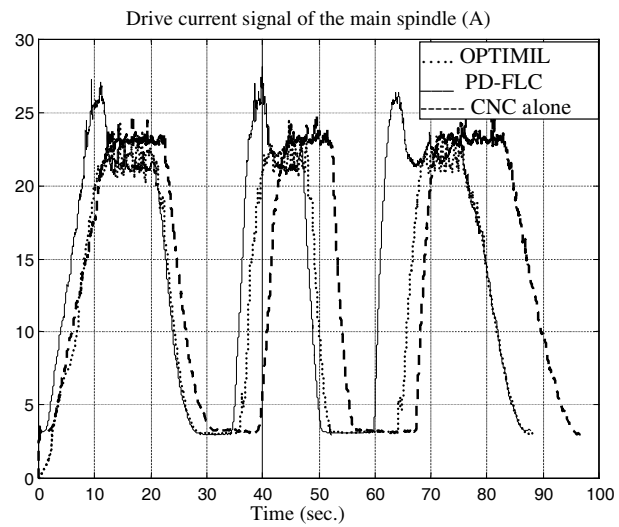

Fig. 6. Time responses of the control systems

\section{Conclusion}

The results of transferring technology to a machine-tool manufacturer through cooperation with a technological center show the effectiveness of a fuzzy control system for dealing with the nonlinear behavior of the machining process. The embedded fuzzy controller is able to work using only internal CNC signals. Moreover, it can run in parallel with other applications without any synchronization problems. However, future work is necessary to refine the fuzzy controller's performance and so to improve the transient response.

All this work, from hypothesis to implementation and experimentation, including design and analysis, is done following the classical patterns. Our focus at all times lay on the practical implementation, so that the results we have presented in this work are for a real life industrial plant. The results show that internal CNC signals can double as an intelligent, sensorless control system. Actual industrial tests show a higher machining efficiency: in-process time is reduced by $10 \%$ and total estimated savings provided by installing the system are about $78 \%$.

\section{References}

1. Koren Y.: Control of machine tools. Journal of Manufacturing Science and Engineering 119 (1997) 749-755

2. Lauderbaugh L.K., Ulsoy A. G.: Model reference adaptive force control in milling. ASME Journal Engineering of Industry 111 (1989) 13-21

3. Rober S.J., Shin Y.C., Nwokah O.D.I.: A digital robust controller for cutting force control in the end milling process. Journal of Dynamic Systems, Measurement and Control 119 (1997) 146-152

4. Haber R.E., Peres C.R., Alique A., Ros S., Alique J.R.: Towards intelligent machining: hierarchical fuzzy control for end milling process. IEEE Transactions on Control Systems Technology 6 (2) (1998) 188-199 
5. Lin L.-C, Lee G.-Y.: Hierarchical fuzzy control for C-axis of CNC turning centers using genetic algorithms. Journal of Intelligent and Robotic Systems: Theory and Applications 25(3) (1999) 255-275

6. Liu Y., Zhuo L., Wang C.: Intelligent adaptive control in milling process. International Journal of Computer Integrated Manufacturing 12(5) (1999) 453-460

7. Liu Y., Wang C.: Neural networks based adaptive control and optimization in milling process. International Journal of Advanced Manufacturing Technology 15 (1999) 791-795

8. Altintas Y.: Prediction of cutting forces and tool breakage in milling from feed drive current measurements. ASME Journal of Engineering of Industry 11 (1992) 386-392

9. Kim T., Kim P.: Adaptive cutting force control for a machining centre by using indirect cutting force measurements. International Journal of Machine Tool Manufacturing 36(8) (1996) 925-937

10. Yager R., Filev D.: Essentials of Fuzzy Modeling and Control, (New York, John Wiley\&Sons) pp. 313-354 (1994)

11. Ying, H.: Analytical structure of a typical fuzzy controllers employing trapezoidal input fuzzy sets and nonlinear control rules. Information Science 116 (1999) 177-203

12. Fagor Automation: Needs of New Numerical Controls (in Spanish). Producción Mecánica 3 (1999) 74-83

13. Siemens AG: Sinumerik 840D, OEM-package NCK, software release 4, user's manual, Erlangen 1999.

14. Nosrat A.: Adaptive controls lead to peak efficiency, American Machinist (1997) 78-80 